Original paper

\title{
Sociodemographic Influences on Public Interest in Natech Risk Information: Insights from Japan and S. Korea
}

\author{
Dimitrios Tzioutzios ${ }^{1}$ and Ana Maria $\mathrm{Cruz}^{2 *}$
}

Received: 31/12/2020 / Accepted: 17/06/2021 / Published online: 08/10/2021

\begin{abstract}
Disaster risk communicators have long contemplated the significance of sociodemographic dimensions in better understanding and characterising an audience's perceptions. Indeed, various societal and personal factors have been considered as predictors of individual risk attitudes, perceptions and behaviours for an array of hazard types. However, such risk communication issues have only recently started to be explored within the emerging field of conjoint natural and technological disasters, called Natech. In this context, delineating the sociodemographic profile of individuals and appreciating the implications of these aspects on Natech risk communication can assist risk managers in tailoring effective risk communication strategies. This study investigates, among other items, the effects of residents' gender, age, household size, income and educational level on their perceptions of information disclosure concerning Natech risk. The approach draws upon the framework of the Situational Theory of Problem Solving in an attempt to conceptualise the complex issue of information deficiency. Taking into account individuals' situational perception elements, the research focuses on certain cross-situational, sociodemographic features that serve as external, determining factors that shape their problem-solving motivation. Data has been collected from households near industrial parks in Osaka and Kobe in Japan and Yeosu, Suncheon, Gwangyang and Ulsan in S. Korea. The results of our regression analysis indicated mostly weak and insignificant effects, except for gender and age that suggested negative and positive influences to individuals' communicative attitudes, respectively. The implications of the institutional differences between the two countries are also discussed within the sphere of chemical and Natech risk communication.
\end{abstract}

Keywords: Risk communication, Natech, Risk information disclosure, Situational theory, Sociodemographic factors

\footnotetext{
${ }^{1}$ Department of Urban Management, Graduate School of Engineering, Kyoto University

${ }^{2}$ Disaster Prevention Research Institute, Kyoto University

* Corresponding author email: cruznaranjo.anamaria.2u@kyoto-u.ac.jp
} 


\section{INTRODUCTION}

Over the years, research scholars have highlighted the exchange of information between experts and communities as the sine qua non for stakeholder involvement and risk governance (Renn and Walker 2008; Aven and Renn 2010; Renn and Klinke 2013), and particularly so in the context of industrial, nuclear and environmental accidents (Shapiro 2005; Palenchar 2008). Risk communication and information disclosure have recently started to attract more academic attention in the Natech accident risk discourse (Figueroa 2013; Suarez-Paba et al. 2020). Natech constitutes a special type of event that entails technological accidents triggered by a natural hazard and involves the release of hazardous materials (Cruz and Suarez-Paba 2019; Suarez-Paba et al. 2019; UNDRR-APSTAAG 2020). They are considered low-probability but high-consequence events with severe impacts on the regional population, environment and economy. Typical examples of Natech include the Fukushima nuclear accident following the Great East Japan Earthquake and Tsunami (GEJET) 2011 (Cruz and Krausmann 2013) and the oil spills that occurred during Hurricanes Katrina and Rita (Cruz and Krausmann 2009).

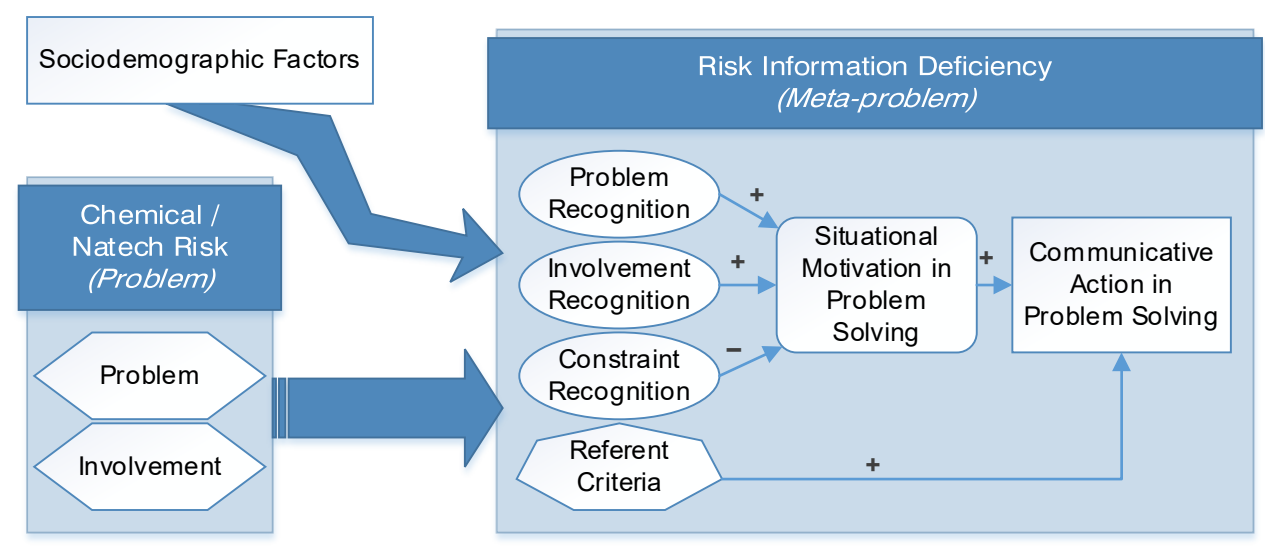

Figure 1. Conceptualisation of Sociodemographic Determinants and Risk Information Deficiency

Disclosure of hazard and risk information allows communities to enhance their preparedness against potential disasters through sharing crucial information with the public. Also, disclosure paves the way for the civic discourse about the decision-making processes involved in risk management (Gutteling and Wiegman 1996; Aven and Renn 2010). Analysing this idea within the specific context of chemical and Natech accidents, Figure 1 describes two kinds of 'problems' from the perspective of risk communication. On the one hand, individuals perceive the underlying Natech accident risk itself as the initial problem that directly or indirectly affects their lives to various perceived degrees. This issue is typically the subject of risk perception studies that explore how risk is socially understood and experienced (Wachinger and Renn 2010). On the other hand, if chemical and Natech risk communication is limited-or even nonexistent - individuals may find themselves lacking necessary hazard or risk information about 
the potential accidents, which is otherwise crucial for their effective preparedness and response to a potential event. In this regard, information deficiency presents a secondary problem that directly stems from the initial accident risk. Individuals then form their own perceptions about this issue in terms of its acknowledged severity, personal views, associated challenges and so forth.

Kim and Grunig (2011) approached this problematic situation from a public relations and mass communications standpoint, defining this discrepancy as a cognitive meta-problem that follows the initial perceptual problem: 'one's perception that something is missing and that there is no immediately applicable solution to it' (p. 128). This conceptualisation presents a unique opportunity to study the issue of Natech risk information deficiency through the lens of Situational Theory ${ }^{3}$. Even though perception about this meta-problem is not the same as the Natech risk perception per se, it can be argued that they are related: the higher the concern about a potential Natech accident, the more salient the issue of information deficiency becomes to the individual. The argument is that by utilising the interpretative framework provided by the Situational Theory of Problem Solving (STOPS) (J.-N. Kim and Grunig 2011), risk communicators can identify and understand the behaviour of publics with increased communicative activeness. These types of publics are more likely to diligently search for, review and synthesise available information and, furthermore, reciprocate the efforts and engage in two-way communication (Grunig and Kim 2017). Therefore, focusing on communicative actors allows risk managers to learn more about the community's demands, fears and (mis-)perceptions concerning both the risk information deficiency and the underlying risk in order to effectively address them and take their interests into consideration in the decision-making process (Grunig 2018).

Furthermore, developing an effective methodology to identify and categorise stakeholder groups has been the Holy Grail and a recurring topic of debate among risk communication and perception researchers for several decades. Creating a reliable segmentation method aims to tailor risk communication strategies to targeted audiences for the purposes of increasing a communication plan's efficacy and maximising the yield of invested resources. In this context, sociocultural and demographic factors have frequently come under scrutiny as potentially potent and robust predictors of individuals' risk attitudes, perceptions and behaviours for an array of natural and technological hazard types (e.g., Savage 1993; Sund et al. 2017; for a review see Wachinger and Renn 2010). Hence, sociodemographic influences have been widely regarded as valuable inputs for disaster risk managers and policy-makers in their quest to understand and predict disaster risk perception.

\footnotetext{
${ }^{3}$ The Situational Theory posits that publics can be identified and segmented from a larger population according to the level of activeness or passiveness of their communication behaviour. Grunig conceptualised the original Situational Theory of Publics (STP) in the ' 60 s as a framework to understand when people communicate and what is the role of information in their decisions. Kim and Grunig (2011) then introduced the Situational Theory of Problem Solving (STOPS) as an extension and generalisation of STP that explains why and how individuals communicates during a problematic situation. For a detailed review of the historical development of the situational theory, interested readers are referred to Kim and Grunig (2011) and Kim and Krishna (2014).
} 
Nonetheless, this risk perception research is still in its infancy with very limited studies so far within the emerging field of Natech communication (Cruz and Suarez-Paba 2019). Yu et al. (2017) was one of the first studies to focus on households' Natech risk perception and evacuation behaviour during the GEJET 2011 around an oil refinery complex in Sendai, Japan. Demographic characteristics did not prove to be strong predictors of Natech risk perception based on their findings, but the authors proposed that further investigation is required. Quite recently, Slack et al. (2020) investigated hazard perceptions in conjunction with institutional trust vis-à-vis Hurricane Harvey in 2017 by surveying households along the Texas Gulf Coast, of the United States. They utilised demographic attributes rather as supplementary controls for their primary research focusing on institutional distrust, but - as with the aforementioned study - their model coefficients did not suggest strong effects.

Given the little research available and considering the inconclusive results of previous studies on the subject of sociodemographic determinants of Natech risk perception, we follow a novel approach that is based on a communicative framework (for reasons explained above), rather than on the Psychometric Paradigm (see e.g., Slovic et al. 1981; Slovic 2000) or the Cultural Theory of Risk (see e.g., Douglas and Wildavsky 1982; Breakwell 2007) that have been used so far. Our main research question is: Do sociodemographic factors influence citizens' situational perception and motivation to communicate with each other about the issue of Natech risk information deficiency? More specifically, we venture to explore whether and how households' sociodemographic characteristics affect their perceptions of the metaproblem of chemical and Natech risk information deficiency. By doing so, we intend to shed some light on the influence of sociodemographic variables on situational perception elements about this issue.

In a basic attempt to reveal any underlying institutional parameters, we looked at households near industrial complexes in two countries that share a relatively similar sociocultural background: Japan and South Korea. The cultural dimensions of Hofstede (2001) (i.e., collectivism/individualism and masculinity/femininity) are used to support our argument about the similarities of the two societal structures in terms of their organisational cultures (see e.g., G. H. Hofstede 2001; House et al. 2004; G. Hofstede et al. 2010). Without disregarding, of course, the discrepancies when comparing the superordinate cultural groups (Park et al. 2016), the predominant characteristic of Japan and S. Korea generally appears to be collectivism. However, Japan seems to score higher on masculinity in contrast to the rather feminist organisational culture of S. Korea (S. H. Kim and Kim 2016). Perhaps more important for our purposes though is the fact that Japan and S. Korea bear a stark difference in terms of chemical and Natech risk communication. S. Korea consolidated and updated its risk management and communication regulations with respect to technological accidents under a comprehensive law titled Chemical Controls Act. A recent amendment refined existing fragmented and outdated articles as well as introduced more detailed provisions regarding public disclosure of chemical information (Ministry of Environment, Republic of Korea 2018). In contrast, despite its deep and advanced disaster education culture regarding natural hazards and regardless of international trends (e.g., Sendai Framework for DRR - UNISDR 2015), Japan has yet to 
introduce any specific regulatory framework that includes standards for public disclosure of information related to chemical risks. Based on this situation, we selected the two countries for our study to explore any effects of the differences in the regulatory frameworks that govern chemical and Natech risk communication on the situational variables under study.

The rest of this article is organised as follows. Section 2 offers a brief overview of the perceptual and cognitive elements of STOPS used in our approach, and then summarizes the findings of previous research on the influence of sociodemographic determinants on risk perception. Section 3 presents the research hypotheses and data collection methods. Section 4 describes the multivariate analysis methods employed. Section 5 includes the analysis results, while Section 6 synthesizes and discusses the key research findings and considers policy implications. The final section offers a summary, and considers the study's limitations and future research prospects.

\section{LITERATURE REVIEW}

\subsection{Perceptual and Cognitive Variables of Situational Motivation}

Situational approaches that use latent constructs (e.g., individual behaviour, cognition) have proven advantageous in analysing the dynamic nature of publics (J.-N. Kim, Ni, and Sha 2008; Chon 2019). This research endeavour employs the four perceptual and cognitive variables of the Situational Theory of Problem Solving (STOPS), namely Problem Recognition (PR), Constraint Recognition (CR), Involvement Recognition (IR) and Referent Criterion (RC), along with Situational Motivation in Problem Solving (SM) (J.-N. Kim and Grunig 2011), to address the issue of Natech risk information deficiency. Researchers have used the situational variables in the past to effectively identify communicatively active public segments ( $\mathrm{Ni}$ and Kim 2009; Y. Kim et al. 2016), as well as to plan communication strategies and predict individuals' communicative behaviours (Chon 2019).

STOPS posits that individuals assume a communicatively active behaviour in terms of acquiring, selecting and transmitting information when they become committed to problem solving (J.-N. Kim and Grunig 2011). This Communicative Action in Problem Solving (CAPS) is dictated by the individual's situational motivation along with any available referent criteria, that is, past experiences, subjective knowledge or expectations applicable to the issue (see Figure 1 for a visual representation). Situational motivation is conceptualised in turn as the product of the three antecedents; problem, involvement and constraint recognition. The first element refers to the perceived severity of the situation, the second to the perceived personal relationship with the problematic situation, and the last to the perceived barriers that limit one's ability to communicate about the problem (J.-N. Kim and Grunig 2011; J.-N. Kim and Krishna 2014). Translating STOPS into the context of Natech risk communication, individuals perceive the meta-problem stemming from the risk information deficiency, their personal connection 
with it and the challenges that limit their ability to take action to resolve it. Based on their knowledge, subjective judgmental rules (e.g., moral or cultural issues) and expectations about how Natech risk information should be handled, their situational motivation drives them to engage in communicative action.

Finally, STOPS has been successfully applied in multiple fields, including health communication and post-incident public relation crisis communication among others (J.-N. Kim and Krishna 2014). Its potential only begun to be explored in the field of chemical risk communication. Furthermore, it has been used in various sociocultural settings, including Asian countries and particularly S. Korea (J.-N. Kim et al. 2012; Chon 2019).

\subsection{Sociodemographic Factors and Risk Perception}

Our attention focuses on cross-situational elements, namely sociodemographic features of involved citizens, as external influential factors that may — or may not—shape the individual's situational perception and problem-solving motivation. Since studying the individuals' opinions concerning the issue of risk information disclosure so systematically is a relatively novel endeavour, the demographic determinants of situational perception have not been thoroughly documented and understood yet. At first glance, the topic may be examined from a public relations perspective focusing on the motivation to communicate and its situational antecedents, while an approach based on risk perception is also warranted due to the specific nature of the issue.

In the former case, researchers working with Situational Theory examined sociodemographic factors that were hypothesised to define individuals' situational perception towards a given problem, with rather modest results. Kim et al. (2012), for example, tested the effects of gender, age, education level and income on individuals' situational perception for topics that receive mass media coverage only to find a significant, moderate influence of age on reducing problem and involvement recognition. Correspondingly, Lovari et al. (2012) studied the influence of similar demographics on situational perception for civic issues (i.e., unemployment, safety, transportation), finding a statistically significant association and small effect between age and problem recognition. This time, a positive direction was found, meaning that age slightly increased problem recognition. As a general rule, Situational Theory academics have argued that cross-situational variables, such as demographic characteristics of individuals, do not exert any substantial effects in comparison to the situational factors (Grunig 1997; J.-N. Kim et al. 2009; 2012), which has also been confirmed in the aforementioned studies.

Perhaps more interesting, however, is the fact that the specific origin of the problem under question is intrinsically tied to risk perception about the underlying chemical and Natech accident risk. This rationale explains why it is important to reconsider the effects of sociodemographic factors on situational perception in the context of risk. Actually, the 
literature about the influence of key demographic characteristics on risk perception is plentiful. Researchers introduced a wide range of contributing factors from physical attributes (e.g., gender and age) to socioeconomic aspects (e.g., education, marital status and income level). Nonetheless, the results were not always definitive, while even the direction of some relationships depended on the particular case study.

Possibly the most studied demographic determinant is gender, as Rowe and Wright (2001, 384) remark. A consistent motif in the risk perception studies is that males typically tend to regard risks as smaller and less problematic compared to females (Savage 1993; Slovic 1999). This finding was confirmed in the case of man-made hazards, namely radioactive waste disposal and global warming (Davidson and Freudenburg 1996). Later research also supported this finding across various types of risk, including natural hazards among others (Sund et al. 2017), or with reference to disaster preparedness measures for floods (Cvetković et al. 2018). Nonetheless, findings have not always revealed an increased risk perception by women. For example, Slack et al. (2020) observed that gender had no statistically significant influence on an individual's level of worry about future impacts of tropical storms. Several researchers noted the ambiguity of this factor's effect on risk perception for a variety of natural hazards, ranging from volcanic and seismic to hydro-meteorological. Their respective analyses revealed that previous exposure to such hazards was actually the underlying cause of fluctuation in risk perception levels (Kunz-Plapp and Werner 2006; Barberi et al. 2008; Wachinger and Renn 2010).

Another demographic characteristic commonly included in risk perception studies is age. Despite the number of studies looking at the relationship between these two aspects, major inconsistencies seem to emerge from the findings (Wachinger and Renn 2010; Cvetković et al. 2018; D. K. D. Kim and Madison 2020; Slack et al. 2020). For instance, one study on singlefamily homeowners residing in Florida found that age was actually correlated with reduced hurricane risk perceptions (Peacock et al. 2005). Furthermore, Huang et al. (2012), reported an association between older individuals and lower risk perception in the case of Hurricane Ike, and reasoned that perhaps older people anticipated smaller personal impact from that particular hazard. On the other hand, other researchers, such as Sjöberg (2004), demonstrated a positive correlation between older individuals and risk associated with nuclear waste. It is clear that consensus has not yet been reached on this matter. As far as Natech risk is concerned, researchers pointed out that older persons residing near an industrial area in Sendai reported higher degrees of concern about their lives and properties being affected by an accident following the Great East Japan Earthquake, even though age - the study noted — did not appear to be a persistent and strong determinant (Yu et al. 2017).

Concerning the effects of individuals' educational attainment on risk perception, Wachinger et al. (2013) concluded that people with different educational levels show differences in risk perception regarding natural hazards. Other studies suggest an inverse correlation between the two: highly educated people demonstrated decreased perceived risk (Savage 1993; Rowe and Wright 2001; Sund et al. 2017; Cvetković et al. 2018; D. K. D. Kim and Madison 2020) for 
various risk domains, including natural hazards. Nonetheless, there have been instances where research efforts failed to discover any significant correlations between risk perception and level of education (Sjöberg 2004). Sundblad et al. (2007) also considered the potential effects of education attainment on risk perception about climate change, arguing that a higher educational level may increase a person's sense of control and therefore reduce perceived risk. This seems to be the case in terms of chemical hazards, as well. One study found evidence to associate higher education with less concern about chemical risk and more favourable attitudes towards related technologies (Kraus, 1992 cited in Rowe and Wright 2001), while another study revealed that higher educational level reduced the perceived risk associated with radioactive waste disposal (Flynn et al. 1993 cited in Rowe and Wright 2001). Lastly, Sund et al. (2017) noted that education could also be connected to individuals placing excessive value on the "correctness" of probabilities and consequences of potential accidents, which in turn may increase or decrease the associated perceived risks based on the level of risk misperception within the general population. Considering the particular topic of this research, however, it is noteworthy that Kim and Madison (D. K. D. Kim and Madison 2020) identified a positive correlation between educational level and information-seeking efficacy in terms of flood risk.

Furthermore, income has been proposed as an additional influential sociodemographic factor, but once again the findings have been mixed. Lower income levels have been linked to an increase in risk perception for technological and natural hazards in the past (Savage 1993). Fothergill and Peek (2004) suggested that individuals with lower income have elevated risk perceptions because of restricted control, potential technological ignorance, lack of social integration that provides them access to risk communication mechanisms, and amplified fear of losing their houses and livelihoods. More recent studies also support this inverse correlation between risk perception and income level in the context of natural hazards (Sund et al. 2017; D. K. D. Kim and Madison 2020). Nonetheless, there have been cases where a significant association between these factors could not be supported by the results (Sjöberg 2000; Cvetković et al. 2018). While a few studies have pointed to a significant relationship between income and risk perception (Donner and Rodríguez 2008; M. K. Lindell and Hwang 2008), the literature review conducted by Wachinger and Renn (2010) led them to conclude that economic factors generally (with the exception of homeownership) do not seem to have a significant influence either on risk perception or willingness to adopt preparedness measures.

Household size was also tested as a determining factor of risk perception and protective action. In the context of tropical storms, larger families showed decreased likelihood of evacuating their houses (Dash 2002). Later research also confirmed this inverse relationship between household size and evacuation likelihood with statistically significant, strong predictors, but revealed that household composition might play an important role as well (Dash and Gladwin 2007). In detail, larger households with children showed a higher propensity to evacuate during a hurricane, while larger households with elderly members were less likely to do so (Dash and Gladwin 2007; Solis et al. 2009). Such a negative relationship between family size and hurricane evacuations in Florida was demonstrated by Solis et al. (2009), although results were statistically insignificant. In the context of Natech risk, however, Yu et al. (2017), 
in their study on the evacuation behaviour following the oil refinery explosion, could not find any significant evidence to either support or reject this correlation.

One of the - admittedly-less-investigated factors in risk perception research is having dependents in the household. Kim and Madison (2020) expected a positive relationship between risk perception and families with dependents, based on the premise that it is within human nature for care-givers to feel a greater responsibility to protect those they take care of (be it children or elderly). Nonetheless, they could not find any statistically significant results to support this hypothesis when investigating the 2016 Louisiana floods in the United States. Moreover, Solis et al. (2009) examined the influence of the number of children and the existence of pets on household evacuation behaviour. Their statistically significant results suggested that households with more children were more likely to evacuate during major hurricanes in 2005 in Florida, but the opposite was true for households with pets. As far as marital status is concerned, studies have failed to find any conclusive and statistically significant results to support either a positive or negative correlation (Basolo et al. 2009; Xu et al. 2018). However, there has been evidence suggesting that single individuals are more likely to prepare emergency supplies in case of flood (Cvetković et al. 2018).

As explained above, social and individual factors - aside from gender - do not seem to play a significant role but may act as mediators or amplifiers between the connections of risk perception, public trust and disaster preparedness (Wachinger et al. 2013). Yet, the interest in the correlation between sociodemographic variables and risk perception still grows, despite the unfruitful research efforts so far. Lindell (2013) acknowledges that, although these variables continue to be unreliable predictors, their potential in helping experts better understand stakeholders' risk perception is great. If nothing else, it is exactly these inconclusive research findings that fuel interest in discovering any underlying linkages between individual sociodemographic aspects and risk perception. This interest is particularly strong in the emerging field of Natech risk, where the influence social and individual factors exert on people's risk perception and protective actions has only recently started to be examined (e.g. Yu et al. 2017; Slack et al. 2020).

\section{METHODOLOGY}

\subsection{Research Aim and Hypotheses}

This study explores whether and how households' sociodemographic characteristics affect their perceptions about the meta-problem of Natech risk information deficiency. Japan and S. Korea are both highly industrialised countries, with some of their largest industrial complexes being located along their eastern coastlines. S. Korea-unlike Japan-is not located in a seismically active region, and the geomorphology of the greater region may be protecting it from potential devastating tsunamis, yet both countries are subject to large tropical storms 
originating from the Pacific Ocean, heavy rainfalls and landslides almost at a yearly rate. Hence, it can be argued that both countries are at a relatively high risk of Natech accidents happening.

As explained earlier, the main research aim is as follows: Do sociodemographic factors influence citizens' situational perception and motivation to communicate with each other about the issue of Natech risk information deficiency? Considering the infancy of Natech risk communication and perception research and the novelty of the STOPS framework in the risk communication field, this study adopts an exploratory approach. Its modest aim is to investigate at an introductory level the sociodemographic influences on situational variables. Thus, the focus is primarily on developing hypotheses for future work. Specifically, this research investigates eight factors: gender, age, educational level, annual household income level, the existence of a spouse, of children and finally, and whether the respondent lived in Japan or S. Korea.

For the purposes of this study, a 'positive effect' on the situational variables is conceptualised as a contribution to the factors that in turn increase the individuals' communicative activeness concerning the meta-problem of Natech risk information deficiency. In detail, an increase in the variables of situational motivation (along with its respective antecedents) and referent criteria is considered as positive. It should be noted that, due to the intrinsic negative aspect of Constraint Recognition, such a 'positive influence' on Constraint Recognition from any sociodemographic factor is hypothesised as an inverse relationship between the two variables and vice versa.

The following assumptions are formed based on the literature review from the standpoint of risk perception. Males are likely to underestimate risks; therefore, the subsequent information deficiency problem is not regarded as prevalent, which is hypothesised to reduce the individual's communicative activeness about it. Moreover, older citizens have been facing the risk and the associated information deficiency problem for longer, and thus, they are expected to perceive it as more severe, but find it more difficult to resolve it. Similarly, education is anticipated to increase individuals' awareness about the risk and subsequently about the lack of information. However, higher levels of education may also provide the means to do something about the problem. Additionally, the larger the household is, the more individuals are exposed to the risk; hence, the perceived situation is hypothesised to be exacerbated. Conversely, a higher income level means more resources are available to effectively cope with the situation, so the problem is not expected to be perceived as large. Hypotheses are similar in the case of the existence of a spouse and children. Having a (co-)dependent household member is anticipated to increase concerns about the meta-problem without delineating any immediately available solution to it, which in turn impels communicative action. Finally, Koreans are expected to be comparatively less concerned about the meta-problem because they have a regulatory framework about chemical risk information disclosure in effect. The chemical risk communication mechanisms in place are hypothesised to provide some ideas about how this issue could be resolved, while reducing the perceived challenges and inviting communication. 
Table 1. Research Hypotheses

\begin{tabular}{|c|c|c|c|c|c|c|c|c|}
\hline Variable & Gender & Age & Education & Income & H. Size & Spouse & Children & Country \\
\hline$P R$ & $H 1_{a}:-$ & $H 2_{a}:+$ & $H 3_{a}:+$ & $H 4_{a}:-$ & $H 5_{a}:+$ & $H 6_{a}:+$ & $H 7_{a}:+$ & $H 8_{a}:-$ \\
\hline$I R$ & $H 1_{b}:-$ & $H 2_{b}:+$ & $H 3_{b}:+$ & $H 4_{b}:-$ & $H 5_{b}:+$ & $H 6_{b}:+$ & $H 7_{b}:+$ & $H 8_{b}:-$ \\
\hline$C R$ & $H 1_{c}:+$ & $H 2_{c}:+$ & $H 3_{c}:-$ & $H 4_{c}:-$ & $H 5_{c}:-$ & $H 6_{c}:-$ & $H 7_{c}:-$ & $H 8_{c}:-$ \\
\hline$R C$ & $H 1_{d}:-$ & $H 2_{d}:+$ & $H 3_{d}:+$ & $H 4_{d}:-$ & $H 5_{d}:+$ & $H 6_{d}:+$ & $H 7_{d}:+$ & $H 8_{d}:+$ \\
\hline$S M$ & $H 1_{e}:-$ & $H 2_{e}:+$ & $H 3_{e}:+$ & $H 4_{e}:-$ & $H 5_{e}:+$ & $H 6_{e}:+$ & $H 7_{e}:+$ & $H 8_{e}:+$ \\
\hline
\end{tabular}

Source: Original work

The arguments above can be formulated in a more operationalised format (Table 1). Gender has a negative effect on Problem Recognition $\left(\boldsymbol{H} \mathbf{1}_{\boldsymbol{a}}\right)$, Involvement Recognition $\left(\boldsymbol{H} \boldsymbol{1}_{\boldsymbol{b}}\right)$, Referent Criteria $\left(\boldsymbol{H} \boldsymbol{1}_{\boldsymbol{d}}\right)$ and Situational Motivation $\left(\boldsymbol{H} \boldsymbol{1}_{\boldsymbol{e}}\right)$, and a positive effect on Constraint Recognition $\left(\boldsymbol{H} \boldsymbol{1}_{\boldsymbol{c}}\right)$. Age has a positive effect on all Problem Recognition $\left(\boldsymbol{H} \boldsymbol{2}_{\boldsymbol{a}}\right)$, Involvement Recognition $\left(\boldsymbol{H} \boldsymbol{2}_{\boldsymbol{b}}\right)$, Constraint Recognition $\left(\boldsymbol{H} \boldsymbol{2}_{\boldsymbol{c}}\right)$, Referent Criteria $\left(\boldsymbol{H} \boldsymbol{2}_{\boldsymbol{d}}\right)$ and Situational Motivation $\left(\boldsymbol{H} \mathbf{2}_{\boldsymbol{e}}\right)$. Education has a positive effect on Problem Recognition $\left(\boldsymbol{H} \mathbf{3}_{\boldsymbol{a}}\right)$, Involvement Recognition $\left(\boldsymbol{H} \boldsymbol{3}_{\boldsymbol{b}}\right)$, Referent Criteria $\left(\boldsymbol{H} \boldsymbol{3}_{\boldsymbol{d}}\right)$ and Situational Motivation $\left(\boldsymbol{H} \boldsymbol{3}_{\boldsymbol{e}}\right)$, and a negative effect on Constraint Recognition $\left(\boldsymbol{H} \boldsymbol{3}_{\boldsymbol{c}}\right)$. Income has a negative effect on all Problem Recognition $\left(\boldsymbol{H} \boldsymbol{4}_{\boldsymbol{a}}\right)$, Involvement Recognition $\left(\boldsymbol{H} \mathbf{4}_{\boldsymbol{b}}\right)$, Constraint Recognition $\left(\boldsymbol{H} \mathbf{4}_{c}\right)$, Referent Criteria $\left(\boldsymbol{H} \boldsymbol{4}_{\boldsymbol{d}}\right)$ and Situational Motivation $\left(\boldsymbol{H} \boldsymbol{4}_{\boldsymbol{e}}\right)$. Household size has a positive effect on Problem Recognition $\left(\boldsymbol{H 5 _ { a }}\right)$, Involvement Recognition $\left(\boldsymbol{H 5 _ { b }}\right)$, Referent Criteria $\left(\boldsymbol{H \boldsymbol { 5 } _ { \boldsymbol { d } }}\right)$ and Situational Motivation $\left(\boldsymbol{H} \boldsymbol{5}_{\boldsymbol{e}}\right)$, and a negative effect on Constraint Recognition $\left(\boldsymbol{H} \boldsymbol{5}_{\boldsymbol{c}}\right)$. Likewise, the existence of a spouse has a positive effect on Problem Recognition (H6 $\mathbf{a}$ ), Involvement Recognition $\left(\boldsymbol{H} \boldsymbol{6}_{\boldsymbol{b}}\right)$, Referent Criteria $\left(\boldsymbol{H} \boldsymbol{6}_{\boldsymbol{d}}\right)$ and Situational Motivation $\left(\mathbf{H} \mathbf{6}_{\mathbf{e}}\right)$, and a negative effect on Constraint Recognition $\left(\boldsymbol{H} \boldsymbol{\sigma}_{\boldsymbol{c}}\right)$, and children has a positive effect on Problem Recognition $\left(\boldsymbol{H} \mathbf{7}_{\boldsymbol{a}}\right)$, Involvement Recognition $\left(\boldsymbol{H} \mathbf{7}_{\boldsymbol{b}}\right)$, Referent Criteria $\left(\mathbf{H} \mathbf{7}_{\mathbf{d}}\right)$ and Situational Motivation $\left(\boldsymbol{H} 7_{\boldsymbol{e}}\right)$, and a negative effect on Constraint Recognition $\left(\mathbf{H} 7_{\mathbf{c}}\right)$. Finally, the country of residence has a negative effect on Problem Recognition $\left(\boldsymbol{H} \boldsymbol{8}_{\boldsymbol{a}}\right)$, Involvement Recognition $\left(\boldsymbol{H} \boldsymbol{\delta}_{\boldsymbol{b}}\right)$ and Constraint Recognition $\left(\boldsymbol{H} \boldsymbol{\delta}_{\boldsymbol{c}}\right)$, and a positive effect on Referent Criteria $\left(\boldsymbol{H} \boldsymbol{\delta}_{\boldsymbol{d}}\right)$ and Situational Motivation $\left(\boldsymbol{H} \boldsymbol{8}_{\boldsymbol{e}}\right)$.

\subsection{Data Collection}

Data were collected via self-administered, anonymous household questionnaire surveys. A seven-point Likert-type scale ranging from $1=$ 'Strongly Disagree' to 7 = 'Strongly Agree' was used to code the responses for the situational variables. At least three items per latent construct were included as a rule. The wording of the questions was based on measurement items tested and validated in previous applications of STOPS (J.-N. Kim et al. 2012; Chen et 
al. 2017) with small adjustments where needed (see Table 2 for the survey measurement items). The initial version of the questionnaire was reviewed by a panel of 30 experts. After minor modifications to the items other than the verified STOPS measures, the questionnaire was translated by bilingual experts from English into Japanese and Korean, while a back-translation verified its effectiveness.

Table 2. STOPS Measurement Items

\begin{tabular}{|c|c|}
\hline $\begin{array}{l}\text { Problem } \\
\text { statement }\end{array}$ & $\begin{array}{l}\text { There is a lack of publicly available information about potential chemical } \\
\text { accidents at the industrial park in the area. }\end{array}$ \\
\hline \multirow{3}{*}{$\begin{array}{l}\text { Problem } \\
\text { Recognition } \\
\text { (PR) }\end{array}$} & PR1 I think this is an important problem. \\
\hline & Government institutions should take action to solve this problem. \\
\hline & $\begin{array}{l}\text { Concerning this problem, I think there is a large gap between the way } \\
\text { PRings should be and the way they are now. }\end{array}$ \\
\hline \multirow{3}{*}{$\begin{array}{l}\text { Problem } \\
\text { Recognition } \\
\text { (IR) }\end{array}$} & IR1 This problem could have serious consequences for me. \\
\hline & This problem could make a difference in my daily life. \\
\hline & There is a strong relationship between myself and this problem. \\
\hline \multirow{2}{*}{$\begin{array}{l}\text { Constraint } \\
\text { Recognition } \\
\text { (CR) }\end{array}$} & CR1 I believe I can improve the situation regarding this problem. \\
\hline & $\begin{array}{l}\text { CR2 My opinions matter to those in the government, who are working on this } \\
\text { problem. }\end{array}$ \\
\hline \multirow{3}{*}{$\begin{array}{l}\text { Referent } \\
\text { Criterion } \\
\quad(\mathrm{RC})\end{array}$} & RC1 I have a clear idea about how to deal with this problem. \\
\hline & RC2 I have an idea about how the government should approach this problem. \\
\hline & $\begin{array}{l}\text { RC3 I believe there are examples from other regions in Japan on how to deal } \\
\text { with this problem. }\end{array}$ \\
\hline \multirow{3}{*}{$\begin{array}{l}\text { Situational } \\
\text { Motivation } \\
\quad(\mathrm{SM})\end{array}$} & SM1 I am curious about this problem. \\
\hline & SM2 I frequently think about this problem. \\
\hline & I want to better understand this problem. \\
\hline
\end{tabular}

Source: Original work based on Kim et al. 2012; Chen, Hung-Baesecke, and Kim 2017

With the intent of collecting data from individuals exposed to a potential Natech accident, households within $2 \mathrm{~km}$ from industrial installations were selected for this study. Areas with prominent industrial parks, neighbouring residential districts were targeted in both countries, specifically districts in Higashinada (Kobe) and Sakai-Senboku (Osaka) in Japan, and Yeosu, Suncheon, Gwangyang and Ulsan in S. Korea. For the Japanese sample, 2,630 questionnaires were distributed using post mail services resulting in $N=330^{4}$ responses ( $12.47 \%$ response rate). Participation was completely voluntary without any financial incentive. The Korean sample $(N=300 ; 100 \%$ response rate) was collected via an online survey employing the Tillion panel, the largest survey panel in the country, using locational restrictions. Participation was again

\footnotetext{
${ }^{4} 327$ valid replies after discarding 2 unanswered questionnaires and 1 unengaged respondent (i.e., answered ' 7 ' throughout the questionnaire).
} 
voluntary, but a small financial compensation in the form of promotional coupons was provided to participants. Data collection periods were January 26-March 8 in 2018 for the Japanese sample and March 9-18, 2020 for the Korean.

\section{ANALYSIS}

The Japanese dataset required treatment for the missing values. The 327 responses were reduced to 317 , after passing the Little's MCAR test, by excluding respondents whose questionnaire fill-out rate did not reach $90 \%$. The remaining dataset was tried again using Little's MCAR test, justifying data imputation for the observed variables of each latent construct with the respective variable median ${ }^{5}$ (Hair et al. 2010). The resulting Japanese dataset was then joined with the Korean. Preliminary reliability analysis for the combined dataset $(N=617)$ exhibited robust latent construct validity for the five situational variables, as demonstrated by the Cronbach's $\alpha$ values that were above the .60 threshold. In detail: PR, $\alpha=.839$; IR, $\alpha=.857$; CR, $\alpha=.601$ after the exclusion of 1 problematic item; RC, $\alpha=.718$; and $\mathrm{SM}, \alpha=.812$. Next, the values for each of the five situational variables were calculated as the mean of their respective constituent, observed variables. Finally, listwise deletion was employed once again, this time based on the responses to the demographic variables, in order to produce the final dataset for the regression analyses.

Prior to building the regression models, a check for any multicollinearity among the independent variables was performed. Variance inflation factors (VIFs) were smaller than 2.51 across all models, which is well under the threshold of 5 that would point to any serious problems of multicollinearity. Moderate, statistically significant correlations, nonetheless, were observed between country and age $(-.507, p<0.01)$, education $(-.381, p<0.01)$, household size $(-.370, p<0.01)$, spouse $(-.349, p<0.01)$ and children $(-.462, p<0.01)$, when correlation analysis was performed on the sociodemographic determinants. According to Belsley et al. (1980), fairly high statistical correlations among predictors are not always problematic in regression analysis. In this vein, predictors were included despite their significant, moderate correlation in order to control for them in the respective models. DurbinWatson tests indicated no signs of dependency between observations for any model. Preliminary tests displayed no issues of non-linearity or homoskedasticity for any of the five models, suggesting thus that model specification could progress.

A series of Multiple Linear Regression analyses was conducted in order to test the formulated hypotheses and assess the effects - or lack thereof - of the sociodemographic factors on each of the situational variables. One model for each of the five dependent, situational variables was specified. In each model, all factors were entered in order to account

\footnotetext{
${ }^{5}$ This technique is not expected to alter the variable mean (Hair et al. 2010), since the percentage of missing information per variable did not exceed $2.3 \%$ (suggested threshold $10 \%$ ).
} 
for their effects regardless of the statistical significance of their respective coefficients. The general model is given by the expression as follows:

$$
Y_{\text {SitVar }}=f(\text { Gender }, \text { Age, Education, Income, Household Size, Spouse, Children, Country })
$$

\section{RESULTS}

First, the demographic profile for the Japanese and Korean samples was delineated with respect to their gender, age, level of education, annual household income level, number of household members, whether they had a spouse and children (Table 3). Even though at first glance the ratio of the Japanese and Korean sub-categories of the dataset seems to be wellbalanced (a decent 55/45), a more detailed examination of their compositions reveals that the Japanese and Korean sub-categories of the dataset are quite different. Indeed, while almost 3 in 5 Japanese respondents were male, females comprise the majority in the Korean sub-group. The discrepancy becomes more prevalent concerning age. Almost $60 \%$ of the respondents are above 60 years old among Japanese respondents, whereas Korean respondents are no older than 59. Similarly, all Korean respondents had at least graduated from a vocational/technical school in contrast to Japanese $40 \%$ of whom had finished only elementary and high school education. Considering the annual household income level, the groups appeared to be fairly similar, with only slightly more Koreans identifying themselves in the middle tier. The remaining three categories display significant divergencies. Households of Japanese respondents seem to be much smaller comparatively, with only $20 \%$ of them exceeding 3 members, while more than half of the Korean households have at least 4 members. Additionally, $70 \%$ of Japanese respondents had a spouse compared to $96.8 \%$ of Koreans. Finally, a similar situation was observed with respect to children living in the household; almost 9 out of 10 Korean households had children, compared to only 4 in 10 within the Japanese group.

Concerning the five situational variables under study, Table 4 offers a brief overview of the descriptive statistics for the combined dataset. Bearing in mind that the midpoint of the 7-point scale used in this study was 4 , the following can be deduced. First, problem and involvement recognition both have rather elevated means (i.e. 5.8 and 5.3 respectively), while their values of Skewness and Kurtosis suggest peaks at higher values-particularly so for PR with a comparatively smaller $\sigma$, too. The values for the other three variables indicate more equally distributed responses. Two more points are worth noting here. Referent criteria was the only variable with positive skewness, while the only variable embedded with a negative meaning, constrained recognition, also received relatively high responses. 
Table 3. Summary of Sample Characteristics

\begin{tabular}{|c|c|c|c|c|}
\hline \multirow{3}{*}{ Category } & \multicolumn{2}{|c|}{ Country } & \multirow{2}{*}{\multicolumn{2}{|c|}{ Total }} \\
\hline & \multirow{2}{*}{$\begin{array}{c}\text { Japan } \\
54,85 \% \\
\end{array}$} & \multirow{2}{*}{$\begin{array}{c}\text { S. Korea } \\
45,15 \% \\
\end{array}$} & & \\
\hline & & & 485 & $100 \%$ \\
\hline \multicolumn{5}{|c|}{ Gender } \\
\hline Female (1) & $41,35 \%$ & $53,42 \%$ & 227 & $46,80 \%$ \\
\hline Male (2) & $58,65 \%$ & $46,58 \%$ & 258 & $53,20 \%$ \\
\hline \multicolumn{5}{|c|}{ Age } \\
\hline 19 or younger $(1)$ & $0,38 \%$ & $0,00 \%$ & 1 & $0,21 \%$ \\
\hline $20-29(2)$ & $1,88 \%$ & $2,74 \%$ & 11 & $2,27 \%$ \\
\hline $30-39(3)$ & $10,15 \%$ & $26,48 \%$ & 85 & $17,53 \%$ \\
\hline $40-49(4)$ & $11,28 \%$ & $34,25 \%$ & 105 & $21,65 \%$ \\
\hline $50-59(5)$ & $16,92 \%$ & $36,53 \%$ & 125 & $25,77 \%$ \\
\hline $60-74(6)$ & $42,11 \%$ & $0,00 \%$ & 112 & $23,09 \%$ \\
\hline 75 or older $(7)$ & $17,29 \%$ & $0,00 \%$ & 46 & $9,48 \%$ \\
\hline \multicolumn{5}{|c|}{ Educational Level } \\
\hline Elementary School (1) & $2,63 \%$ & $0,00 \%$ & 7 & $1,44 \%$ \\
\hline High School (2) & $36,47 \%$ & $0,00 \%$ & 97 & $20,00 \%$ \\
\hline Vocational / Technical School (3) & $15,04 \%$ & $21,00 \%$ & 86 & $17,73 \%$ \\
\hline Bachelor Degree (4) & $37,22 \%$ & $73,52 \%$ & 260 & $53,61 \%$ \\
\hline Master Degree / PhD (5) & $8,65 \%$ & $5,48 \%$ & 35 & $7,22 \%$ \\
\hline \multicolumn{5}{|c|}{ Annual Household Income Level } \\
\hline Low $(0)$ & $38,35 \%$ & $15,07 \%$ & 135 & $27,84 \%$ \\
\hline Middle (1) & $34,96 \%$ & $57,53 \%$ & 219 & $45,15 \%$ \\
\hline High (2) & $26,69 \%$ & $27,40 \%$ & 131 & $27,01 \%$ \\
\hline \multicolumn{5}{|c|}{ Household Size } \\
\hline 1 Member (1) & $19,17 \%$ & $1,83 \%$ & 55 & $11,34 \%$ \\
\hline 2 Members (2) & $38,35 \%$ & $21,00 \%$ & 148 & $30,52 \%$ \\
\hline 3 Members (3) & $23,68 \%$ & $26,48 \%$ & 121 & $24,95 \%$ \\
\hline 4 Members (4) & $13,16 \%$ & $40,18 \%$ & 123 & $25,36 \%$ \\
\hline 5 Members (5) & $4,14 \%$ & $10,05 \%$ & 33 & $6,80 \%$ \\
\hline 6 Members (6) & $1,13 \%$ & $0,46 \%$ & 4 & $0,82 \%$ \\
\hline 7 or more $(7)$ & $0,38 \%$ & $0,00 \%$ & 1 & $0,21 \%$ \\
\hline \multicolumn{5}{|c|}{ Spouse } \\
\hline No $(0)$ & $30,08 \%$ & $3,20 \%$ & 87 & $17,94 \%$ \\
\hline Yes (1) & $69,92 \%$ & $96,80 \%$ & 398 & $82,06 \%$ \\
\hline \multicolumn{5}{|c|}{ Children } \\
\hline No $(0)$ & $58,27 \%$ & $13,24 \%$ & 184 & $37,94 \%$ \\
\hline Yes (1) & $41,73 \%$ & $86,76 \%$ & 301 & $62,06 \%$ \\
\hline
\end{tabular}


Table 4. Descriptive Statistics of the Situational Variables

\begin{tabular}{|l|c|c|c|c|}
\hline \multicolumn{1}{|c|}{ Situational Variable } & Mean & Std. Deviation & Skewness $^{\text {I }}$ & Kurtosis $^{2}$ \\
\hline Problem Recognition (PR) & 5.82 & .97 & -1.095 & 1.747 \\
\hline Involvement Recognition (IR) & 5.29 & 1.16 & -.761 & .557 \\
\hline Constraint Recognition (CR) & 4.36 & 1.30 & -.250 & -.454 \\
\hline Referent Criteria (RC) & 3.77 & 1.20 & .164 & -.446 \\
\hline Situational Motivation (SM) & 4.63 & 1.20 & -.465 & .162 \\
\hline $\begin{array}{l}{ }^{1} \text { Std. Error }=0.111 \text { and }^{2} \text { Std. Error }=0.221 . \\
N=485\end{array}$
\end{tabular}

Source: Original work

The results from the regression analyses for the five models are summarised next. Looking at the last two columns of Table 5, it becomes apparent that models $R_{1}$ and $R_{2}$ for predicting problem and involvement recognition are not statistically significant at the $95 \%$ confidence interval. Hence, conclusions concerning the influence of any of the sociodemographic factors on these two situational variables (i.e., $\boldsymbol{H} \boldsymbol{1}_{\boldsymbol{a}}$ through $\boldsymbol{H} \boldsymbol{2}_{\boldsymbol{e}}$ ) cannot be confidently drawn. Furthermore, the interpretative power of all models is quite small, even considering relatively low explanatory power standards typically found in psychological research (see Cvetković et al. 2018). $R^{2}$ values range from .047 to .12 , meaning that the best performing model $R_{4}$ accounts only for $12 \%$ of the total variance observed in the referent criteria.

Table 5. Regression Model Fit Results Summary

\begin{tabular}{|l|l|c|c|c|c|c|}
\hline Model & \multicolumn{1}{|c|}{ Situational Variable } & $\mathbf{R}$ & $\mathbf{R}^{\mathbf{2}}$ & $\mathbf{R}^{\mathbf{2}} \mathbf{a d j}$. & $\mathbf{F}(8,476)$ & $\boldsymbol{p}$ \\
\hline$R_{1}$ & Problem Recognition (PR) & .176 & .031 & .015 & 1.899 & .058 \\
\hline$R_{2}$ & Involvement Recognition (IR) & .175 & .031 & .014 & 1.886 & .060 \\
\hline$R_{3}$ & Constraint Recognition (CR) & .303 & .092 & .077 & 6.034 & .000 \\
\hline$R_{4}$ & Referent Criteria (RC) & .346 & .120 & .105 & 8.081 & .000 \\
\hline$R_{5}$ & Situational Motivation (SM) & .218 & .047 & .031 & 2.964 & .003 \\
\hline$N=485$ & \multicolumn{7}{|l}{} \\
\hline
\end{tabular}

Source: Original work

Moving on to the regression coefficients (Table 6), we observed that none of the estimated coefficients for education, income, household size, having a spouse or having children is statistically significant in any model. Therefore, hypotheses $H 3_{c}$ through $H 7_{c}, H 3_{d}$ through $H 7_{d}$ and additionally $H 3_{e}$ through $H 7_{e}$ cannot be confidently confirmed or rejected on account of insignificant evidence. On the other hand, respondents' gender appears to slightly increase constrain recognition $\left(H 1_{c}\right)(B=.34, p<.01)$ and reduce situational motivation $\left(H 1_{c}\right)(B=-.32$, 
the effects of sociodemographic determinants. Instead, our results resonated with the conclusions from previous risk perception studies in the context of Natech (Yu et al. 2017; Slack et al. 2020) and risk communication in general (Wachinger and Renn 2010; Wachinger et al. 2013): effects proved mostly weak and insignificant. In general, the cross-situational impotence of sociodemographic characteristics in comparison to the perceptual variables of STOPS (Grunig 1997; J.-N. Kim et al. 2009; 2012) was confirmed in this study. Nevertheless, our risk communication-oriented approach invites an interesting discussion about the findings.

Specifically, gender seemed to have a positive relationship with constraint recognition and a negative relationship with situational motivation for problem solving. As hypothesised based on the risk perception literature, males appear to be less motivated to communicate about the Natech risk information deficiency issue, while they perceive more obstacles in pursuit of information. Research has shown that males tend to underestimate risks in various contexts (e.g., Savage 1993; Sund et al. 2017), and perhaps this reduced perceived severity of the initial chemical risk is what leads to a subsequently lower cognitive meta-problem appraisal and interest in a solution. This reasoning would explain the negative effect on situational motivation, as this factor is defined by problem, involvement and constraint recognition. Moreover, perhaps the comparatively lower interest of male respondents also magnifies the perceived limitations, simply because individuals have not invested themselves in analysing the issues in order to overcome them.

In terms of the effects of age, our findings seem to align more with the literature that regards age as a dampening factor for risk perception (Peacock et al. 2005; Huang et al. 2012). Along with age, there seems to come experience in how to resolve the meta-problem of Natech risk information deficiency. Older individuals appear to have more referent criteria readily available about how this problem should be handled. Following the conceptualisation of STOPS (J.-N. Kim and Krishna 2014), this may be due to more experiences accumulated over the years, as well as established expectations about how Natech risk information deficiency could be resolved. Of course, there is no established association between Natech risk perception and the communicative behaviour towards the issue of risk information deficiency, yet we could argue that the alleviating effects of reducing perceived limitations in resolving the metaproblem contribute in turn to lessening the perceived severity of the whole situation.

On the other hand, the variable of country demonstrated some interesting findings. Korean respondents appear to perceive themselves as less constrained in resolving the meta-problem of Natech risk information deficiency, whilst they have referent criteria they consider readily applicable to the issue. Results were strong and statistically significant in this regard. There even seems to be a positive, alleviating effect on problem recognition, although conclusions could not be statistically supported. Now, even though our methodological approach does not warrant a cross-cultural comparison at the national level between Japan and S. Korea, we included this factor in our analysis as a control for any underlying institutional parameters, particularly bearing in mind the difference in the chemical risk management regulatory frameworks of the two countries. Although we can only speculate at this point given these 
circumstantial findings, we could entertain the idea that the recently introduced Chemical Controls Act, which includes chemical risk information disclosure provisions, actually succeeds in creating a more inviting chemical and Natech risk communication environment compared to Japan. Korean respondents seem to be more communicatively active towards resolving the issue of Natech risk information disclosure, and perhaps this is due to the existence of a regulatory environment.

Of course, the central point of our reasoning is a fairly similar, collectivistic organisational culture among the two countries (see e.g., G. H. Hofstede 2001; House et al. 2004; G. Hofstede et al. 2010) that does not significantly skew the observed relationships. However, contemplating the arguments about the dissimilarities of the two organisational cultures (S. H. Kim and Kim 2016; Park et al. 2016), a ceteris paribus assumption for the sociocultural parameters at play cannot be upheld. Moreover, any conclusions based on the comparison between the Japanese and Korean sub-groups in our survey respondents must be treated with extra caution considering the sample discrepancies presented in the dataset. Furthermore, we cannot exclude any hidden factors we may have omitted or understated in our approach. Nevertheless, these preliminary results may pave the way for future research that will test more rigorously this argument against various cross-cultural influences.

\section{CONCLUSIONS}

Going beyond risk perception studies that have traditionally approached the relationship between risk communication and sociodemographic determinants through the psychometric and cultural theory paradigms, we borrowed the interpretative framework of STOPS to investigate situational variables instead. We framed the lack of Natech risk information as a cognitive meta-problem that stems from the original, underlying Natech accident risk. Individuals perceive this issue and, according to the purposeful communication narrative of STOPS, become motivated to communicate and overcome it. In this context, this study set to investigate the potential effects of sociodemographic factors in shaping individuals' situational perceptions and communicative behaviour concerning the issue of Natech risk information deficiency. We collected data from households near prominent industrial parks in Osaka and Kobe in Japan, and Yeosu, Suncheon, Gwangyang and Ulsan in S. Korea, to assess the effects of factors such as gender, age, household size, income and educational level. The results of our regression analysis indicated mostly weak and insignificant effects, except for gender and age that suggested negative and positive influences on individuals' communicative attitudes, respectively. The implications of the institutional differences between the two countries were also discussed within the sphere of chemical and Natech risk communication.

As one of the very few studies in the emerging field of Natech risk communication, we hope the findings of this research can contribute to formulating and focusing directions for further investigations. However, there are some drawbacks in this study that should be discussed here 
with an outlook to future research. There was no intention of conducting a cross-cultural study that would involve sociocultural constructs, and would focus on comparing the two samples, even at a national level. Arguably, our approach combined responses from individuals of different sociocultural backgrounds, and so introduced-inevitably-some culture-specific biases. We acknowledge that our efforts to address this issue with the introduction of a single control variable are far from optimal. In this respect, this research topic would greatly benefit from a full-fledged cross-cultural study that would incorporate sociocultural dimensions to effectively capture and disambiguate their influences on the situational variables. Moreover, as pointed out in the results section, we did not optimise our sampling method in pursuit of more representative population samples that would permit generalisations and a comparison at the national level between Japan and S. Korea. In contrast, we opted for rather technical criteria, targeting households under immediate risk from a potential Natech accident at the neighbouring industrial facility. Resource limitations did not permit the implementation of a sampling strategy that would simultaneously control for the location and the demographic profile of respondents, thus resulting in notable demographic discrepancies between the two samples. Hence, future studies are encouraged to investigate the topic using different sampling techniques based on demographic criteria (e.g., stratified sampling), especially if aiming for inter-group comparisons. Finally, our study did not explore the potential influence of survey participation incentives on the quality of responses in an opinion questionnaire about disaster risk communication and perception. Although our study employed both data collection strategies (i.e., incentives and not), one for each sample, our findings do not allow us to draw conclusions concerning this matter. Considering that participation incentives might, hypothetically, affect the representation of certain sub-groups within the sample and-by extension - their perceptions, additional research is needed to further investigate these issues.

\section{REFERENCES}

Aven, Terje, and Ortwin, Renn. 2010. Risk Management and Governance: Concepts, Guidelines and Applications. Risk, Governance and Society. Berlin Heidelberg: SpringerVerlag. //www.springer.com/jp/book/9783642139253.

Barberi, Federica, Matthew Davis, Roberto Isaia, Rosella Nave, and Tullio Ricci. 2008. 'Volcanic Risk Perception in the Vesuvius Population'. Journal of Volcanology and Geothermal Research, April. https://doi.org/10.1016/j.jvolgeores.2007.12.011.

Basolo, Victoria, Laura J. Steinberg, Raymond J. Burby, Joyce Levine, Ana Maria Cruz, and Chihyen Huang. 2009. 'The Effects of Confidence in Government and Information on Perceived and Actual Preparedness for Disasters'. Environment and Behavior 41 (3): 33864. https://doi.org/10.1177/0013916508317222.

Belsley, David A., Edwin Kuh, and Roy E. Welsch. 1980. Regression Diagnostics: Identifying Influential Data and Sources of Collinearity. Wiley.

Breakwell, Glynis M. 2007. The Psychology of Risk. Cambridge: Cambridge University Press. https://doi.org/10.1017/CBO9780511819315. 
Chen, Yi-Ru Regina, Chun-Ju Flora Hung-Baesecke, and Jeong-Nam Kim. 2017. 'Identifying Active Hot-Issue Communicators and Subgroup Identifiers: Examining the Situational Theory of Problem Solving'. Journalism \& Mass Communication Quarterly 94 (1): 12447. https://doi.org/10.1177/1077699016629371.

Chon, Myoung-Gi. 2019. 'Government Public Relations When Trouble Hits: Exploring Political Dispositions, Situational Variables, and Government-Public Relationships to Predict Communicative Action of Publics'. Asian Journal of Communication 0 (0): 1-17. https://doi.org/10.1080/01292986.2019.1649438.

Cruz, Ana Maria, and Elisabeth Krausmann. 2009. 'Hazardous-Materials Releases from Offshore Oil and Gas Facilities and Emergency Response Following Hurricanes Katrina and Rita'. Journal of Loss Prevention in the Process Industries 22 (1): 59-65. https://doi.org/10.1016/j.jlp.2008.08.007.

Cruz, Ana Maria, and Elisabeth Krausmann. 2013. 'Vulnerability of the Oil and Gas Sector to Climate Change and Extreme Weather Events'. Climatic Change 121 (1): 41-53. https://doi.org/10.1007/s10584-013-0891-4.

Cruz, Ana Maria, and Maria Camila Suarez-Paba. 2019. 'Advances in Natech Research: An Overview'. Progress in Disaster Science 1 (May): 100013. https://doi.org/10.1016/j.pdisas.2019.100013.

Cvetković, Vladimir M., Giulia Roder, Adem Öcal, Paolo Tarolli, and Slavoljub Dragićević. 2018. 'The Role of Gender in Preparedness and Response Behaviors towards Flood Risk in Serbia'. International Journal of Environmental Research and Public Health 15 (12): 2761. https://doi.org/10.3390/ijerph15122761.

Dash, Nicole. 2002. 'Decision-Making under Extreme Uncertainty: Rethinking HazardRelated Perceptions and Action'. ProQuest ETD Collection for FIU, January.

Dash, Nicole, and Hugh Gladwin. 2007. 'Evacuation Decision Making and Behavioral Responses: Individual and Household'. Natural Hazards Review 8 (3): 69-77. https://doi.org/10.1061/(ASCE)1527-6988(2007)8:3(69).

Davidson, Debra J., and Wiluam R. Freudenburg. 1996. 'Gender and Environmental Risk Concerns: A Review and Analysis of Available Research'. Environment and Behavior 28 (3): 302-39. https://doi.org/10.1177/0013916596283003.

Donner, William, and Havidán Rodríguez. 2008. 'Population Composition, Migration and Inequality: The Influence of Demographic Changes on Disaster Risk and Vulnerability'. Social Forces 87 (2): 1089-1114. https://doi.org/10.1353/sof.0.0141.

Douglas, Mary, and Aaron Wildavsky. 1982. Risk and Culture: An Essay on the Selection of Technological and Environmental Dangers. 1st ed. University of California Press. https://www.jstor.org/stable/10.1525/j.ctt7zw3mr.

Figueroa, Pablo M. 2013. 'Risk Communication Surrounding the Fukushima Nuclear Disaster: An Anthropological Approach'. Asia Europe Journal 11 (1): 53-64. https://doi.org/10.1007/s10308-013-0343-9.

Fothergill, Alice, and Lori A. Peek. 2004. 'Poverty and Disasters in the United States: A Review of Recent Sociological Findings'. Natural Hazards 32 (1): 89-110. https://doi.org/10.1023/B:NHAZ.0000026792.76181.d9.

Grunig, James E. 1997. 'A Situational Theory of Publics: Conceptual History, Recent Challenges and New Research'. In Public Relations Research: An International 
Perspective, edited by Danny Moss, Toby MacManus, and Dejan Verčič, 3-47. London: International Thomson Business Press.

Grunig, James E. 2018. 'Strategic Behavioral Paradigm'. In The International Encyclopedia of Strategic Communication, by Robert L. Heath and Winni Johansen, 1-6. American Cancer Society. https://doi.org/10.1002/9781119010722.iesc0171.

Grunig, James E., and Jeong-Nam Kim. 2017. 'Publics Approaches to Health and Risk Message Design and Processing'. Oxford Research Encyclopedia of Communication, May. https://doi.org/10.1093/acrefore/9780190228613.013.322.

Gutteling, Jan M., and Oene Wiegman. 1996. Exploring Risk Communication. Advances in Natural and Technological Hazards Research. Springer Netherlands. https://doi.org/10.1007/978-94-017-1523-2.

Hair, Joseph F., Jr, William C. Black, Barry J. Babin, and Rolph E. Anderson. 2010. Multivariate Data Analysis. 7 edition. Upper Saddle River, NJ: Pearson.

Hofstede, Geert H. 2001. Culture's Consequences: Comparing Values, Behaviors, Institutions and Organizations Across Nations. 2nd ed. Thousand Oaks, CA: SAGE.

Hofstede, Geert, Gert Jan Hofstede, and Michael Minkov. 2010. Cultures and Organizations: Software of the Mind, Third Edition. 3rd Edition. New York: McGraw-Hill Education.

House, Robert J., Paul J. Hanges, Mansour Javidan, Peter W. Dorfman, and Vipin Gupta. 2004. Culture, Leadership, and Organizations: The GLOBE Study of 62 Societies. 1st Edition. Thousand Oaks, Calif: SAGE Publications, Inc.

Huang, Shih-Kai, Michael K. Lindell, Carla S. Prater, Hao-Che Wu, and Laura K. Siebeneck. 2012. 'Household Evacuation Decision Making in Response to Hurricane Ike'. Natural Hazards Review 13 (4): 283-96. https://doi.org/10.1061/(ASCE)NH.1527-6996.0000074.

Kim, Do Kyun David, and T. Phillip Madison. 2020. 'Public Risk Perception Attitude and Information-Seeking Efficacy on Floods: A Formative Study for Disaster Preparation Campaigns and Policies'. International Journal of Disaster Risk Science 11 (5): 592-601. https://doi.org/10.1007/s13753-020-00307-5.

Kim, Jeong-Nam, and James E. Grunig. 2011. 'Problem Solving and Communicative Action: A Situational Theory of Problem Solving'. Journal of Communication 61 (1): 120-49. https://doi.org/10.1111/j.1460-2466.2010.01529.x.

Kim, Jeong-Nam, Y. R. Jung, S. C. Park, and M. Dutta. 2009. 'Gossiping Science: Lay Diffusers of Science Knowledge and Information'. In Chicago, IL: Applied Communication Division, National Communication Association.

Kim, Jeong-Nam, and Arunima Krishna. 2014. 'Publics and Lay Informatics: A Review of the Situational Theory of Problem Solving'. Annals of the International Communication Association 38 (1): 71-105. https://doi.org/10.1080/23808985.2014.11679159.

Kim, Jeong-Nam, Lan Ni, Sei-Hill Kim, and Jangyul Robert Kim. 2012. 'What Makes People Hot? Applying the Situational Theory of Problem Solving to Hot-Issue Publics'. Journal of Public Relations Research 24 (2): 144-64. https://doi.org/10.1080/1062726X.2012.62 $\underline{6133}$.

Kim, Jeong-Nam, Lan Ni, and Bey-Ling Sha. 2008. 'Breaking down the Stakeholder Environment: Explicating Approaches to the Segmentation of Publics for Public Relations Research'. Journalism \& Mass Communication Quarterly 85 (4): 751-68. https://doi.org/10.1177/107769900808500403. 
Kim, Seung Hyun, and Sangmook Kim. 2016. 'Social Desirability Bias in Measuring Public Service Motivation'. International Public Management Journal 19 (3): 293-319. https://doi.org/10.1080/10967494.2015.1021497.

Kim, Young, Andrea Miller, and Myoung-Gi Chon. 2016. 'Communicating with Key Publics in Crisis Communication: The Synthetic Approach to the Public Segmentation in CAPS (Communicative Action in Problem Solving)'. Journal of Contingencies and Crisis Management 24 (2): 82-94. https://doi.org/10.1111/1468-5973.12104.

Kunz-Plapp, Tina, and Ute Werner. 2006. 'Understanding Risk Perception from Natural Hazards: Examples from Germany'. In RISK21 - Coping with Risks Due to Natural Hazards in the 21st Century: Proceedings of the RISK21 Workshop, Monte Verità, Ascona, Switzerland, 28 November - 3 December 2004, edited by Walter J. Ammann, Stefanie Dannenmann, and Laurent Vulliet, 21:101-8. CRC Press.

Lindell, Michael. 2013. 'North American Cities at Risk: Household Responses to Environmental Hazards'. In Cities at Risk: Living with Perils in the 21st Century, edited by Helene Joffe, Tiziana Rossetto, and John Adams, 109-30. Advances in Natural and Technological Hazards Research. Dordrecht: Springer Netherlands. https://doi.org/10.1007/978-94-007-6184-1_7.

Lindell, Michael K., and Seong Nam Hwang. 2008. 'Households' Perceived Personal Risk and Responses in a Multihazard Environment'. Risk Analysis 28 (2): 539-56. https://doi.org/10.1111/j.1539-6924.2008.01032.x.

Lovari, Alessandro, Valentina Martino, and Jeong-Nam Kim. 2012. 'Citizens' Relationships with a Municipality and Their Communicative Behaviors in Negative Civic Issues'. International Journal of Strategic Communication 6 (1): 17-30. https://doi.org/10.1080/1553118X.2011.634870.

Ministry of Environment, Republic of Korea. 2018. Chemicals Control Act.

Ni, Lan, and Jeong-Nam Kim. 2009. 'Classifying Publics: Communication Behaviors and Problem-Solving Characteristics in Controversial Issues'. International Journal of Strategic Communication 3 (4): 217-41. https://doi.org/10.1080/15531180903221261.

Palenchar, Michael J. "Risk Communication and Community Right to Know: A Public Relations Obligation to Inform" (2008). School of Advertising and Public Relations Publications and Other Works. Public Relations Journal, January. https://trace.tennessee.edu/utk_advepubs/1.

Park, Junsu, Do-Yeong Kim, and Chong Zhang. 2016. 'Understanding Cross-National Differences in Risk Through a Localized Cultural Perspective'. Cross-Cultural Research 50 (1): 34-62. https://doi.org/10.1177/1069397115609560.

Peacock, Walter Gillis, Samuel David Brody, and Wes Highfield. 2005. 'Hurricane Risk Perceptions among Florida's Single Family Homeowners'. Landscape and Urban Planning, Research on the Built and Virtual Environments, 73 (2): 120-35. https://doi.org/10.1016/j.landurbplan.2004.11.004.

Renn, Ortwin, and Andreas Klinke. 2013. 'A Framework of Adaptive Risk Governance for Urban Planning'. Sustainability 5 (5): 2036-59. https://doi.org/10.3390/su5052036.

Renn, Ortwin, and Katherine D. Walker, eds. 2008. Global Risk Governance: Concept and Practice Using the IRGC Framework. International Risk Governance Council Bookseries. Springer Netherlands. https://doi.org/10.1007/978-1-4020-6799-0. 
Rowe, Gene, and George Wright. 2001. 'Differences in Expert and Lay Judgments of Risk: Myth or Reality?' Risk Analysis 21 (2): 341-56. https://doi.org/10.1111/02724332.212116.

Savage, Ian. 1993. 'Demographic Influences on Risk Perceptions'. Risk Analysis 13 (4): 41320. https://doi.org/10.1111/j.1539-6924.1993.tb00741.x.

Shapiro, Marc D. 2005. 'Equity and Information: Information Regulation, Environmental Justice, and Risks from Toxic Chemicals'. Journal of Policy Analysis and Management 24 (2): 373-98. https://doi.org/10.1002/pam.20094.

Sjöberg, Lennart. 2000. 'Factors in Risk Perception'. Risk Analysis 20 (1): 1-12. https://doi.org/10.1111/0272-4332.00001.

Sjöberg, Lennart. 2004. 'Explaining Individual Risk Perception: The Case of Nuclear Waste'. Risk Management 6 (1): 51-64. https://doi.org/10.1057/palgrave.rm.8240172.

Slack, Tim, Vanessa Parks, Lynsay Ayer, Andrew M. Parker, Melissa L. Finucane, and Rajeev Ramchand. 2020. 'Natech or Natural? An Analysis of Hazard Perceptions, Institutional Trust, and Future Storm Worry Following Hurricane Harvey'. Natural Hazards 102 (3): 1207-24. https://doi.org/10.1007/s11069-020-03953-6.

Slovic, Paul. 1999. 'Trust, Emotion, Sex, Politics, and Science: Surveying the Risk-Assessment Battlefield'. Risk Analysis 19 (4): 689-701. https://doi.org/10.1111/j.15396924.1999.tb00439.x.

Slovic, Paul. 2000. The Perception of Risk. London; Sterling, VA: Earthscan.

Slovic, Paul, Baruch Fischhoff, Sarah Lichtenstein, and F. J. C. Roe. 1981. 'Perceived Risk: Psychological Factors and Social Implications [and Discussion]'. Proceedings of the Royal Society of London. Series A, Mathematical and Physical Sciences 376 (1764): 17-34.

Solis, Daniel, Michael H. Thomas, and David Letson. 2009. 'Determinants of Household Hurricane Evacuation Choice in Florida'. In 2009 Annual Meeting: Selected Papers. Atlanta, Georgia: Southern Agricultural Economics Association. https://econpapers.repec.org/paper/agssaeana/45338.htm

Suarez-Paba, Maria Camila, Mathis Perreur, Felipe Munoz, and Ana Maria Cruz. 2019. 'Systematic Literature Review and Qualitative Meta-Analysis of Natech Research in the Past Four Decades'. Safety Science 116 (July): 58-77. https://doi.org/10.1016/j.ssci.2019.02.033.

Suarez-Paba, Maria Camila, Dimitrios Tzioutzios, Ana Maria Cruz, and Elisabeth Krausmann. 2020. 'Toward Natech Resilient Industries'. In Disaster Risk Reduction and Resilience, edited by Muneta Yokomatsu and Stefan Hochrainer-Stigler, 45-64. Disaster and Risk Research: GADRI Book Series. Singapore: Springer. https://doi.org/10.1007/978-981-154320-3 4 .

Sund, Björn, Mikael Svensson, and Henrik Andersson. 2017. 'Demographic Determinants of Incident Experience and Risk Perception: Do High-Risk Groups Accurately Perceive Themselves as High-Risk?' Journal of Risk Research 20 (1): 99-117. https://doi.org/10.1080/13669877.2015.1042499.

Sundblad, Eva-Lotta, Anders Biel, and Tommy Gärling. 2007. 'Cognitive and Affective Risk Judgements Related to Climate Change'. Journal of Environmental Psychology 27 (2): 97-106. https://doi.org/10.1016/j.jenvp.2007.01.003. 
UNDRR-APSTAAG. 2020. 'Asia-Pacific Regional Framework for NATECH (Natural Hazards Triggering Technological Disasters) Risk Management'. Geneva, Switzerland: United Nations Office for Disaster Risk Reduction - Asia-Pacific Science, Technology and Academia Advisory Group. https://www.undrr.org/publication/asia-pacific-regionalframework-natech-natural-hazards-triggering-technological.

UNISDR. 2015. 'Sendai Framework for Disaster Risk Reduction 2015-2030'. UNISDR/GE/2015-ICLUX EN5000 1st edition. Geneva, Switzerland: United Nations Office for Disaster Risk Reduction.

Wachinger, Gisela, and Ortwin Renn. 2010. 'Risk Perception and Natural Hazards'. CapHazNet WP3 Report. Stuttgart, Germany: DIALOGIK Non-Profit Institute for Communication and Cooperative Research.

Wachinger, Gisela, Ortwin Renn, Chloe Begg, and Christian Kuhlicke. 2013. 'The Risk Perception Paradox--Implications for Governance and Communication of Natural Hazards'. Risk Analysis: An Official Publication of the Society for Risk Analysis 33 (6): 1049-65. https://doi.org/10.1111/j.1539-6924.2012.01942.x.

Xu, Dingde, Li Peng, Shaoquan Liu, and Xuxi Wang. 2018. 'Influences of Risk Perception and Sense of Place on Landslide Disaster Preparedness in Southwestern China'. International Journal of Disaster Risk Science 9 (2): 167-80. https://doi.org/10.1007/s13 753-018-0170-0.

Yu, Junlei, Ana Maria Cruz, and Akihiko Hokugo. 2017. 'Households' Risk Perception and Behavioral Responses to Natech Accidents'. International Journal of Disaster Risk Science 8 (1): 1-15. https://doi.org/10.1007/s13753-017-0116-y. 